MARINE MAMMAL SCIENCE, **(*): ***_***(*** 2015)

(C) 2015 Society for Marine Mammalogy

DOI: $10.1111 / \mathrm{mms} .12280$

\title{
Differences in oscillatory whistles produced by spinner (Stenella longirostris) and pantropical spotted (Stenella attenuata) dolphins
}

Pina Gruden ${ }^{1}$ and Paul R. White, Institute of Sound and Vibration Research, University of Southampton, Highfield, SO17 1BJ, United Kingdom; Julie N. Osw ALD, Bio-Waves Inc., 364 2nd Street, Suite \#3, Encinitas, California 92024, U.S.A.; Yvonne BarkLey, NOAA Pacific Islands Fisheries Science Center, 1845 Wasp Boulevard, Building 176, Honolulu, Hawaii 96818, U.S.A.; Salvatore Cerchio, New England Aquarium, 1 Central Wharf, Boston, Massachusetts 02110, U.S.A.; MARC LAMmers, Hawaii Institute of Marine Biology, PO Box 1346, Kane'ohe, Hawaii 96744, U.S.A.; Simone Baumann-Pickering, Scripps Institution of Oceanography, University of California San Diego, La Jolla, California 92093, U.S.A.

\begin{abstract}
Acoustic recordings of two closely related species, spinner dolphin (Stenella longirostris) and pantropical spotted dolphin (Stenella attenuata), were investigated from four different geographic locations: two in the Central Tropical Pacific, one in the Eastern Tropical Pacific and one in the Indian Ocean. The two delphinid species occur in tropical and warm temperate waters, with overlapping ranges. They produce very similar vocalizations, but at the same time their calls exhibit a certain degree of intraspecific variation among different geographic locations as has been observed in other delphinid species. Oscillatory whistles (whistles with at least two oscillations in their frequency contours) were identified and manually extracted from the recordings. Whistles with four or more maxima (oscillations) occurred only in spinner dolphins and they were present in all geographic regions investigated. In addition, the oscillatory whistles with two and three maxima were significantly more frequent in spinner than in spotted dolphins. The differences in oscillatory whistles for these two species seem to be consistent across study areas and therefore, could be used in addition to other whistle features to help distinguish between them.
\end{abstract}

Key words: oscillatory whistles, whistle, global features, decision rule, dolphin, spinner dolphin, pantropical spotted dolphin, Stenella longirostris, Stenella attenuata, towed hydrophone array.

In recent years, Passive Acoustic Monitoring (PAM) systems have increasingly been used to study cetacean behavior and distribution (Mellinger et al. 2007, Van Parijs et al. 2009, Gerrodette et al. 2011, Kusel et al. 2011). PAM has become an important component in the majority of shipboard cetacean surveys as well as in mitigation strategies, since this type of monitoring is not restricted by daylight and is more robust to poor weather conditions than visual monitoring (Barlow and Taylor

\footnotetext{
${ }^{1}$ Corresponding author (e-mail: pg3g12@soton.ac.uk).
} 
2005, Mellinger et al. 2007, Rankin et al. 2007). An important component of many acoustic surveys is to assign detected vocalizations to a specific species for purposes such as abundance estimation and population assessments (Barlow and Taylor 2005, Van Parijs et al. 2009, Gerrodette et al. 2011).

Marine mammals produce characteristic calls and species identification can be achieved with some degree of confidence for many of them (e.g., Clark and Clapham 2004, Moore et al. 2006, Roch et al. 2007, Oswald et al. 2007, Soldevilla et al. 2008, Gillespie et al. 2013). Odontocetes produce a rich variety of vocalizations, which can be grouped into three broad categories: whistles, echolocation clicks, and burst pulses ( $\mathrm{Au} 2000$ ). All of these signals tend to be high frequency, with most of their energy above $2 \mathrm{kHz}$ (Tyack and Clark 2000). Not all odontocete species whistle, but the majority of delphinid species do produce whistles.

Whistles are narrowband, frequency modulated, tonal sounds with fundamental frequencies generally between 2 and $30 \mathrm{kHz}$ (Lammers et al. 2003). They are typically used in social contexts (Lammers et al. 2003) and as such are likely used to convey details such as individual identity (Janik et al. 2006, King and Janik 2013), behavior state (Herzing 1996), and other important information.

Significant intraspecific variation has been found in whistle characteristics and this has been linked to factors such as the behavioral state of animals, social and population structure, and the environmental heterogeneity (Rendell et al. 1999, Matthews et al. 1999, May-Collado and Wartzok 2008). In addition, a high degree of overlap has been observed in the time and frequency characteristics of the whistles of many species (Matthews et al. 1999, Oswald et al. 2007). A number of factors, such as similarity in the body size (Matthews et al. 1999), have been linked to similarity of acoustic signals. This similarity in the whistle characteristics among species, in addition to the high intraspecific variability can make a whistle-based classification of small delphinids challenging.

Two such species are the spinner dolphin (Stenella longirostris) and the pantropical spotted dolphin (Stenella attenuata), which are closely related cosmopolitan delphinid species with similar body size and are known to occur in the same habitats, occasionally in mixed species groups (Reeves et al. 2002, Psarakos et al. 2003). There are four subspecies of spinner dolphins recognized (Perrin et al. 1991): Stenella longirostris longirostris, $S$. l. orientalis, S. l. centroamericana, and S. l. roseiventris. There are two to four subspecies of pantropical spotted dolphin (Shirihai 2006), but only two have been officially described: Stenella attenuata attenuata and $S$. a. graffmani (Perrin 1975).

Several studies have investigated whistle characteristics of these two species in the Pacific and Atlantic oceans (e.g., Bazua-Duran and Au 2002; Lammers et al. 2003; Oswald et al. 2003, 2007; Camargo et al. 2006). Both species have been found to produce whistles within a similar frequency range between 8 and $18 \mathrm{kHz}$. A large repertoire based on whistle contour shape has been investigated for the Hawaiian spinner dolphin (Bazua-Duran and Au 2002) and has been reported to consist of $44 \%$ chirps (whistles with a duration of less than $300 \mathrm{~ms}$ ), $27 \%$ upsweeps and $11 \%$ sine-shaped whistles (a contour with at least two inflection points). To the best of our knowledge, no such study has been conducted for the pantropical spotted dolphin.

This study investigated the occurrence of specific whistle type, the oscillatory shaped whistles (whistles with at least one and a half cycles and two maxima in their frequency contour), in spinner and pantropical spotted dolphins. To this end acoustic recordings from four different locations were analyzed and the rates of observation for different categories of oscillatory whistles were computed for both species. 


\section{Materials AND Methods}

\section{Study Areas and Data Collection}

Acoustic recordings were collected between 1997 and 2012 during 10 shipboard acoustic line transect surveys and two stationary acoustic boat surveys (Table 1). The data were acquired from four geographical locations: the Hawaiian archipelago, Palmyra Atoll, the Eastern Tropical Pacific (ETP), and Madagascar in the Indian Ocean (Fig. 1). The Hawaiian Islands Cetacean and Ecosystem Assessment Survey (HICEAS 2002, 2010) and the L1997 and L1998 surveys were conducted within the Hawaiian Island Archipelago. The Pacific Islands Cetacean and Ecosystem Assessment Survey (PICEAS 2005, 2012) and the B2006 and B2007 surveys contributed data from Palmyra Atoll. Surveys in the ETP were conducted along the west coast of Mexico south to the territorial waters of Peru (STAR 2000, STAR 2003, and STAR 2006). The Zanj 2010 survey in the southwest Indian Ocean was conducted from a $22 \mathrm{~m}$ sailing vessel along the west coast of Madagascar in the Mozambique Channel between $140 \mathrm{~km}$ south of Toliara in the southwest $\left(24^{\circ} 31.952^{\prime} \mathrm{S}, 43^{\circ} 50.152^{\prime} \mathrm{E}\right)$ and Mahajanga in the northwest $\left(15^{\circ} 35.725^{\prime} \mathrm{S}\right.$, $\left.44^{\circ} 54.682^{\prime} \mathrm{E}\right)$. Detailed descriptions of the study areas for HICEAS, PICEAS, and STAR surveys can be found in Oswald et al. (2007, 2008). The study areas covered by B2006 and B2007 and L1997 and L1998 surveys can be found in BaumannPickering et al. (2010) and Lammers et al. (2003), respectively.

During all surveys, except for L1997 and L1998, the data were obtained using a towed hydrophone array. In L1997 and L1998 data were acquired with a single hydrophone while the vessel was stationary. Details of the acoustic equipment and sampling protocol used during each individual survey are summarized in Table 1.

During all surveys, a team of experienced visual observers actively searched for marine mammals using binoculars and the naked eye. Once marine mammals were sighted or detected by acousticians, visual observers noted the species and group size (determined as the best estimate of the number of animals sighted), along with other information and acoustic recordings were made. An encounter was defined as a period

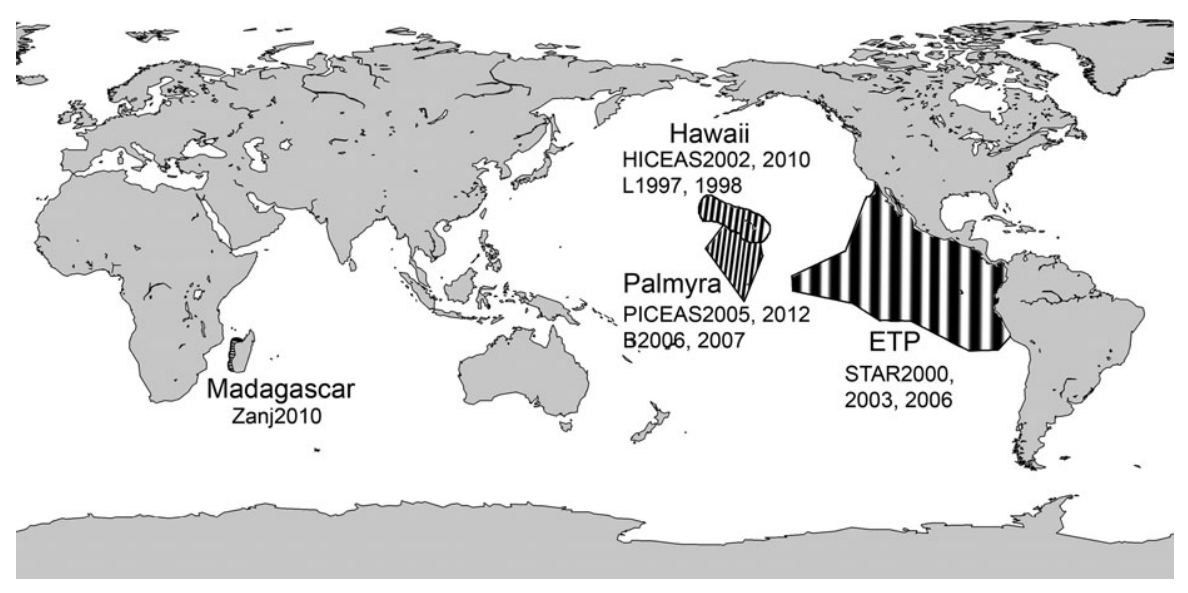

Figure 1. Four study areas, Hawaii, Palmyra, ETP, and Madagascar, with corresponding cruises. 


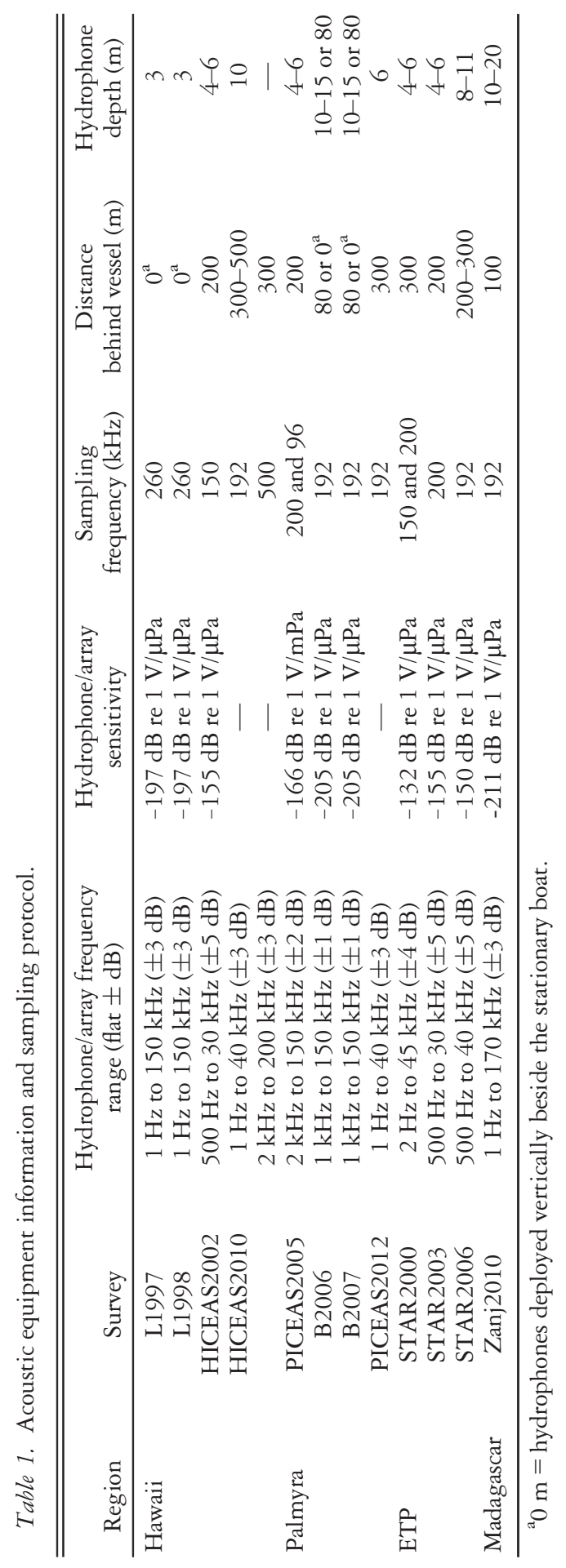


during which the marine mammals were in sight and/or during which they could be monitored acoustically. Only single species encounters of spinner dolphins (S. longirostris) and pantropical spotted dolphins $(S$. attenuata) that were visually confirmed by the observers were included in this study.

\section{Spectrographic and Statistical Analysis}

Where recordings with multiple hydrophone elements were available, the single channel with the best dynamic range and lowest self-noise was selected for the analysis. All data were resampled to have a common sampling frequency of $192 \mathrm{kHz}$. The spectrograms (2,048 point Hanning window, 50\% overlap) of all the recordings were examined manually with Adobe Audition software (version 3.0). All whistles that were comprised of at least one and a half cycles with two maxima (peaks) in their frequency contour were termed oscillatory whistles and were manually extracted from the recordings. ${ }^{2}$ For example, the spinner dolphin whistle in Figure 2 is comprised of one and a half cycles and two maxima, and the pantropical spotted dolphin whistle in the same figure is comprised of two cycles and also two maxima. Oscillatory whistles were placed into one of the three categories; whistles with two maxima, whistles with
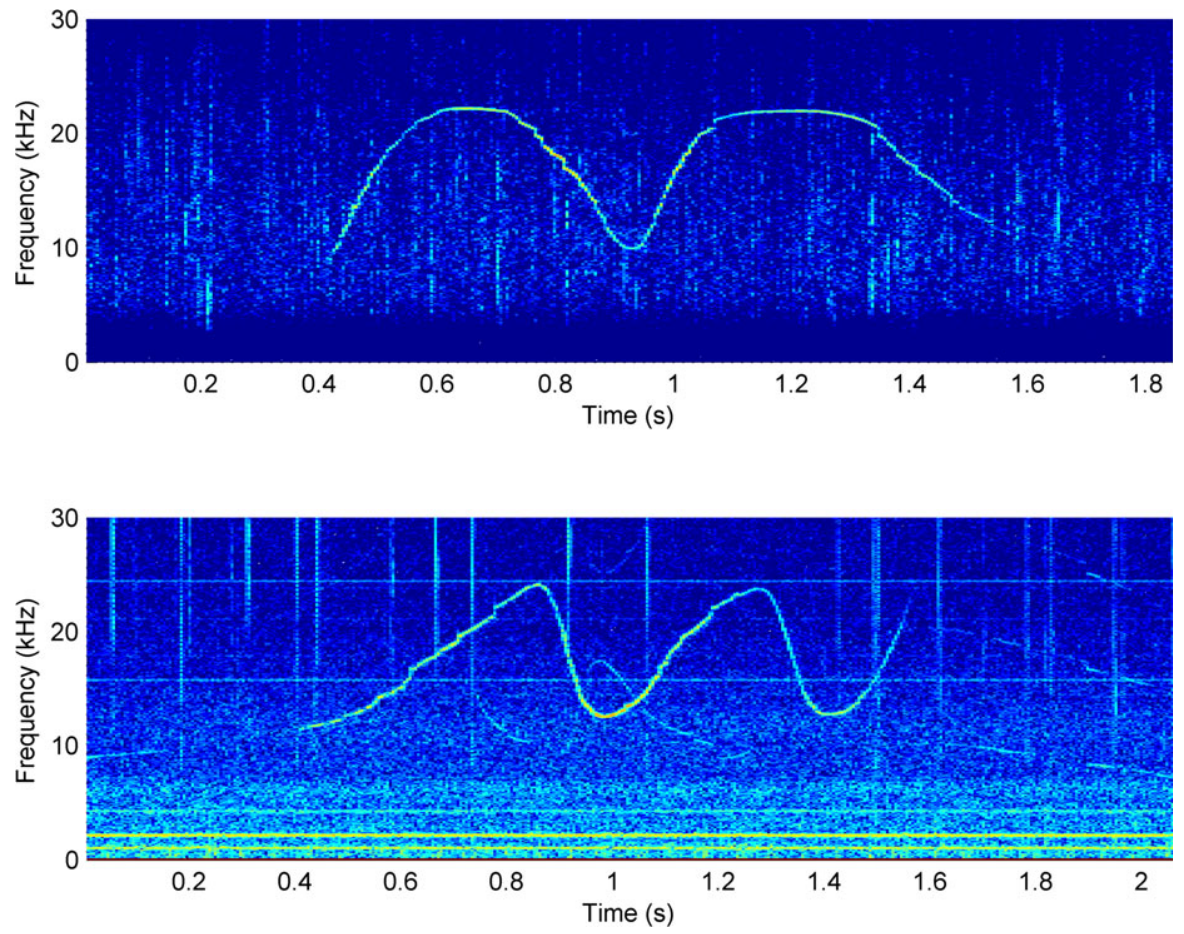

Figure 2. Oscillatory whistle with two maxima (peaks) from spinner (top) and pantropical spotted (bottom) dolphins.

\footnotetext{
${ }^{2}$ The term inflection point (that is often used in describing whistle contours) was avoided due to multiple definitions that make comparisons among studies difficult.
} 
three maxima, or whistles with four or more maxima. The occurrence of each whistle category was investigated in relation to species, group size, region, and encounter duration.

Statistical differences between species for each oscillatory whistle category were evaluated by comparing the rates of occurrence of these whistles. The statistical test employed was based on the ratio of Poisson rates. This technique corrects for different sampling durations that could affect the counts of events and is often applied in biological and medical studies, where data consist of counts of rare events (Selvin 2004, Li et al. 2013). A function (adapted from rateratio.test in R, Fay 2014) was written in MATLAB (version 8.1, MathWorks Inc., Natick, MA, 2013) to compute the ratio of two Poisson rates in order to compare the rates of oscillatory whistles between spinner and spotted dolphins. Rate was computed as the number of whistles per unit time (per minute) and a ratio of two rates was analyzed using a two-sided hypothesis test and confidence intervals were computed as per Fay (2014).

\section{RESULTS}

In total 42 spinner dolphin encounters and 25 pantropical spotted dolphin encounters, with total durations of 833 and 714 min, respectively, were analyzed (Table 2). The median group size for spinner dolphins was 58 animals (interquartile range 70) and the median group size for spotted dolphins was 37 animals (interquartile range 51) (Table 2). The group size was not significantly different between the two species (Wilcoxon rank sum test, tested at 0.05 level of significance, $P=0.18$ ). The group sizes differed between regions and were largest in the ETP and smallest in Madagascar for both species.

The oscillatory whistles were placed in one of the three categories: whistles with two maxima (Fig. 2), three maxima (Fig. 3), and four or more maxima (Fig. 4). Within the same species, individual oscillatory whistles occurred at slightly different center frequencies, had different durations and had slightly different shape (e.g., in terms of frequency bandwidth, sharpness of maxima and steepness of slope), but were categorized based only on the number of maxima.

The oscillatory whistles were found in 57\% (24 out of 42) of spinner dolphin encounters and in 40\% (10 out of 25) of spotted dolphin encounters (Table 2). It should be noted that $79 \%$ of spinner dolphin encounters were very short $(<5 \mathrm{~min})$, whereas less than $1 \%$ of spotted dolphin encounters were shorter than 5 min.

Both species produced whistles with two and three maxima, but only spinner dolphins produced whistles with four or more maxima (Fig. 5). Furthermore, each whistle category occurred significantly (ratio of Poisson rates, tested at 0.05 level of significance) more frequently in recordings of spinner dolphins than in recordings of spotted dolphins (Fig. 5, Table 3).

On the encounter level, it was noted that whistles with two maxima were the most frequently observed and whistles with four or more maxima were the least frequently observed oscillatory whistles for both species (Table 4).

The potential influence of the group size on the oscillatory whistle rate (computed as the number of oscillatory whistles in the encounter divided by the encounter duration) was investigated. When the species was not considered, the correlation between group size and oscillatory whistle rate appeared significant (Spearman rank order correlation coefficient, tested at 0.05 level of significance, $r=0.26, P=0.04$ ). However when correlation was investigated for each species separately, the correlation between 


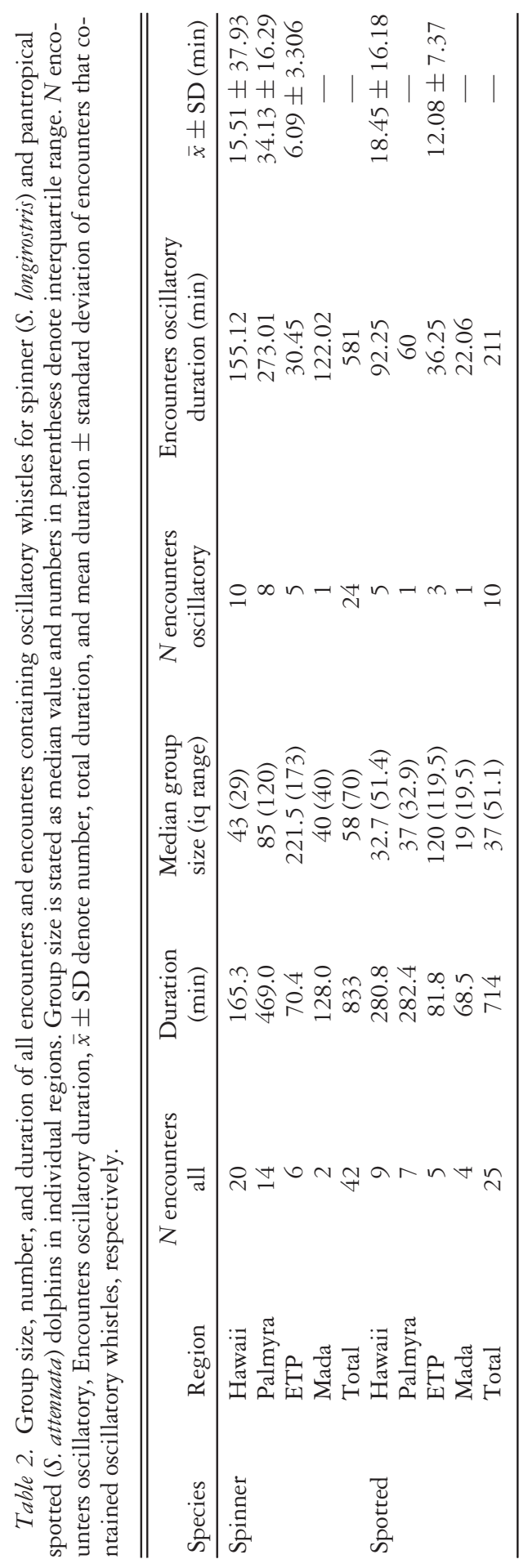



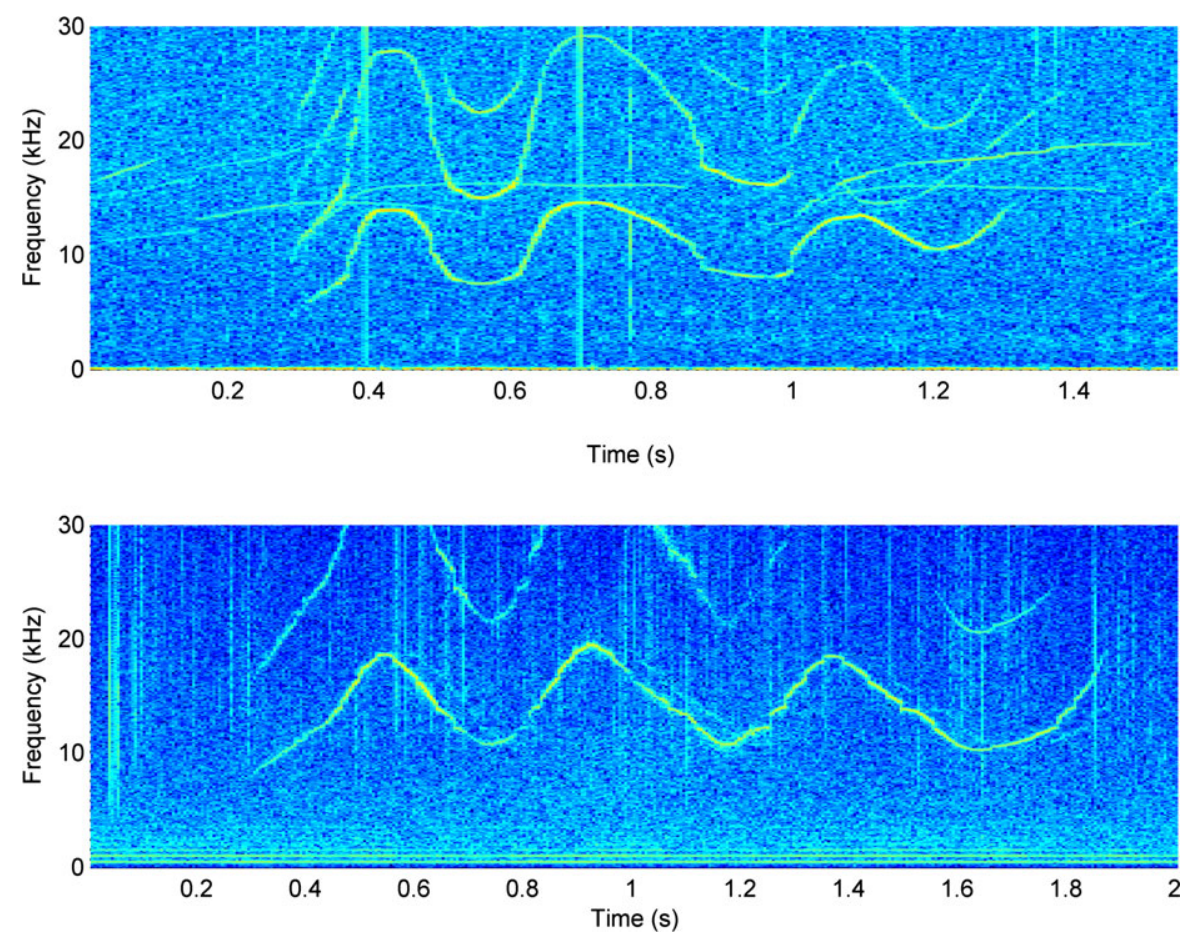

Figure 3. Oscillatory whistle with three maxima (peaks) from spinner (top) and pantropical spotted (bottom) dolphins.

group size and oscillatory whistle rate no longer appeared to be significant (Spearman rank order correlation, tested at 0.05 level of significance, spinner dolphins: $r=0.2$, $P=0.22$; spotted dolphins: $r=0.34, P=0.09$ ).

The occurrence of oscillatory whistles in each geographic region was also investigated (Fig. 6). All oscillatory whistle types occurred in all geographic regions. Oscillatory whistles with four or more maxima were most abundant in the ETP, whistles with three maxima and two maxima were most abundant in Palmyra for spinner dolphins and in Madagascar and Hawaii for spotted dolphins, respectively. Since the encounter duration could have influenced the number of counted whistles, the average oscillatory whistle rate was computed for each region (Fig. 7). Among spinner dolphins, the highest rate of whistles with two maxima and four or more maxima occurred in the ETP and the highest rate of whistles with three maxima occurred in Hawaii. For spotted dolphins the highest rate of whistles with two maxima was in Hawaii and the highest rate for whistles with three maxima in Madagascar. In general the oscillatory whistle rates were higher in spinner than in spotted dolphins in all regions except for Madagascar.

Based on the results above, a simple discrimination rule was developed which could be added to a whistle-based classifier to assist in distinguishing between the two species. This rule was based on the presence or absence of whistles with three and four or more maxima. According to this rule an encounter was classified as spinner dolphin if it contained whistles with three maxima or more, otherwise it was 

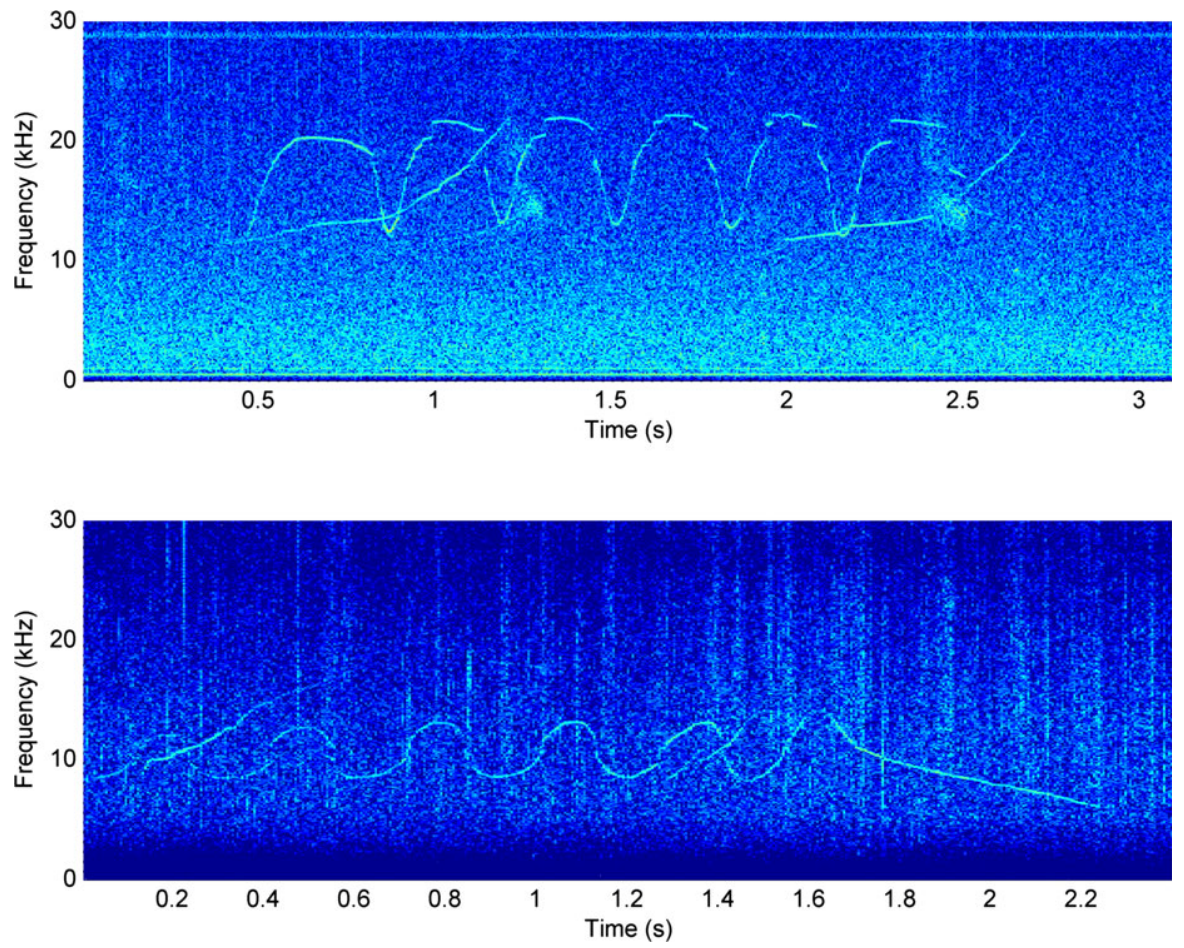

Figure 4. Oscillatory whistle with four or more maxima (peaks) from spinner dolphin from Palmyra (top) and Madagascar (bottom).

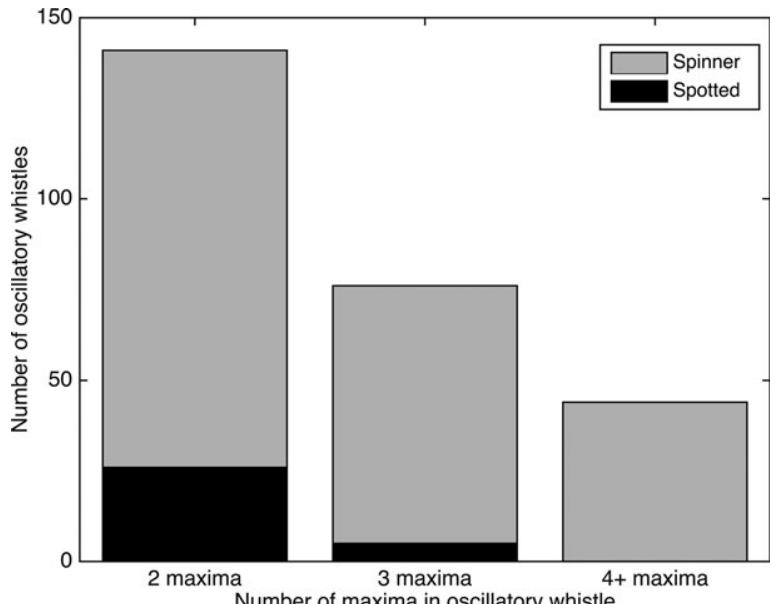

Figure 5. Number of whistles with two, three and four or more maxima for spinner and spotted dolphins. All regions are pooled together. 
Table 3. Ratio of two Poisson rates test for spinner and pantropical spotted dolphin. CINT denotes $95 \%$ confidence interval (lower; upper confidence limit) for rate ratio and $P$ denotes $P$-value. $N$ maxima denotes number of oscillatory whistles with two, three, and four or more maxima.

\begin{tabular}{lccccc}
\hline \hline & Spotted & Spinner & Rate ratio & CINT & $P$ \\
\hline Duration $(\min )$ & 211 & 581 & - & - & - \\
N 2 maxima & 26 & 141 & 0.51 & $0.32 ; 0.78$ & $<0.001$ \\
N 3 maxima & 5 & 76 & 0.18 & $0.06 ; 0.44$ & $<0.001$ \\
N 4+ maxima & 0 & 44 & 0 & $0 ; 0.24$ & $<0.001$ \\
N all & 31 & 261 & 0.33 & $0.22 ; 0.48$ & $<0.001$ \\
\hline
\end{tabular}

Table 4. Percentage of encounters containing oscillatory whistles with two, three, and four or more maxima for spinner and pantropical spotted dolphin when all encounters (All encounters) and just the encounters containing such whistles (Oscill encounters) were taken into account. Numbers in parentheses refer to the number of encounters containing certain whistle category divided by the number of all encounters.

\begin{tabular}{lccccc}
\hline \hline & \multicolumn{2}{c}{ Spinner } & & \multicolumn{2}{c}{ Spotted } \\
\cline { 2 - 3 } \cline { 5 - 6 }$N$ maxima & All encounters & Oscill encounters & & All encounters & Oscill encounters \\
\hline 2 maxima & $48 \%(20 / 42)$ & $83 \%(20 / 24)$ & & $36 \%(9 / 25)$ & $90 \%(9 / 10)$ \\
3 maxima & $43 \%(18 / 42)$ & $75 \%(18 / 24)$ & & $16 \%(4 / 25)$ & $40 \%(4 / 10)$ \\
$4+$ maxima & $26 \%(11 / 42)$ & $46 \%(11 / 24)$ & & - & - \\
\hline
\end{tabular}

classified as spotted dolphin. When this discrimination rule alone was applied to the encounters where oscillatory whistles were detected (24 spinner and 10 spotted dolphin encounters), $83 \%$ of spinner dolphin encounters and $60 \%$ of spotted dolphin encounters were correctly classified.

\section{Discussion}

Oscillatory whistles were identified in the recordings of spinner and pantropical spotted dolphins and their occurrence was explored in relation to species, group size and region.

It was seen that this whistle type occurred significantly more often in spinner than in spotted dolphins and this relationship was found across multiple regions. This infers that there is species-level difference in this vocal behavior that may be useful to aid in species discrimination. Other factors, such as the behavioral state of animals and subspecies present could have influenced the production rate of the oscillatory whistles. Such data were not available for this study and therefore not considered in the analysis. This information could provide further insights into the presence/absence of individual whistle categories and the production rate of oscillatory whistles. However, even without this information, the data sets used in this study are likely representative since they were obtained across different regions, dates, years, and likely behavioral states.

When investigating the effect of the group size on the oscillatory whistle rate, the results were inconclusive. Previous studies on bottlenose dolphins showed that 

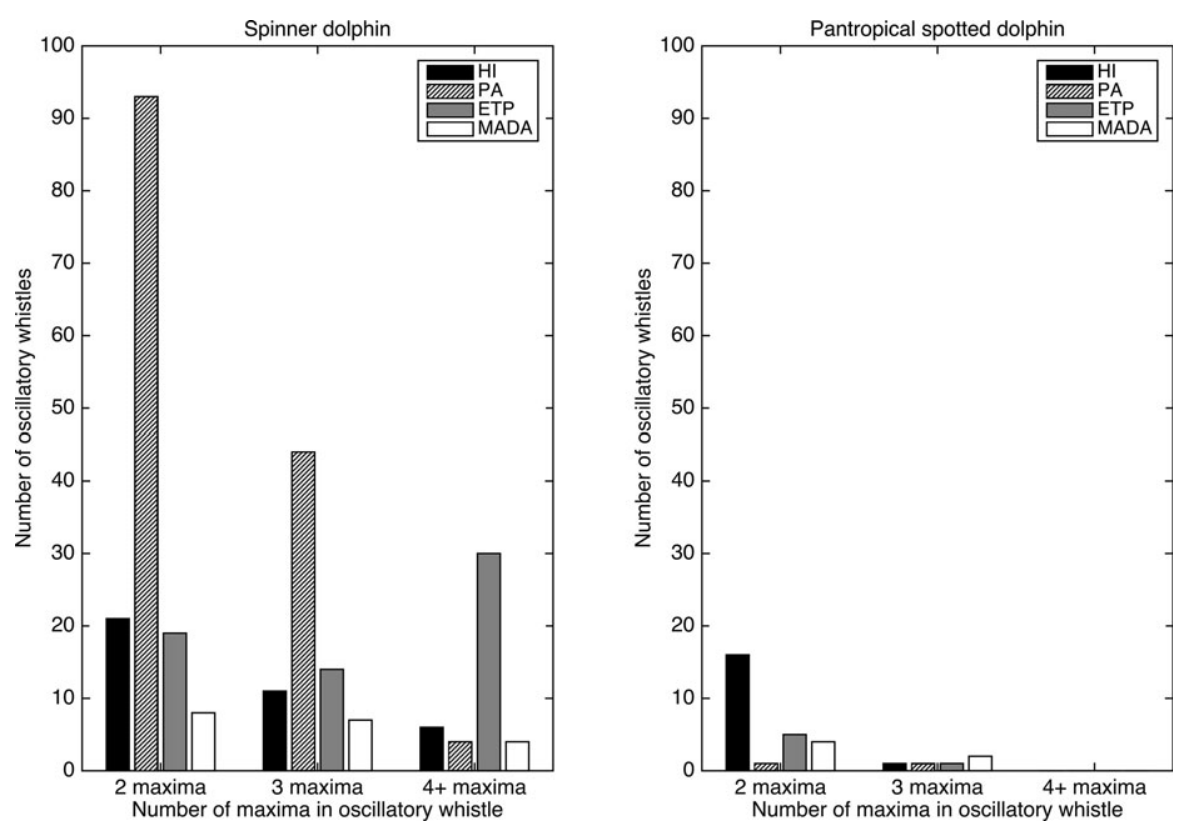

Figure 6. Number of spinner (left) and spotted (right) dolphin oscillatory whistles in each region.
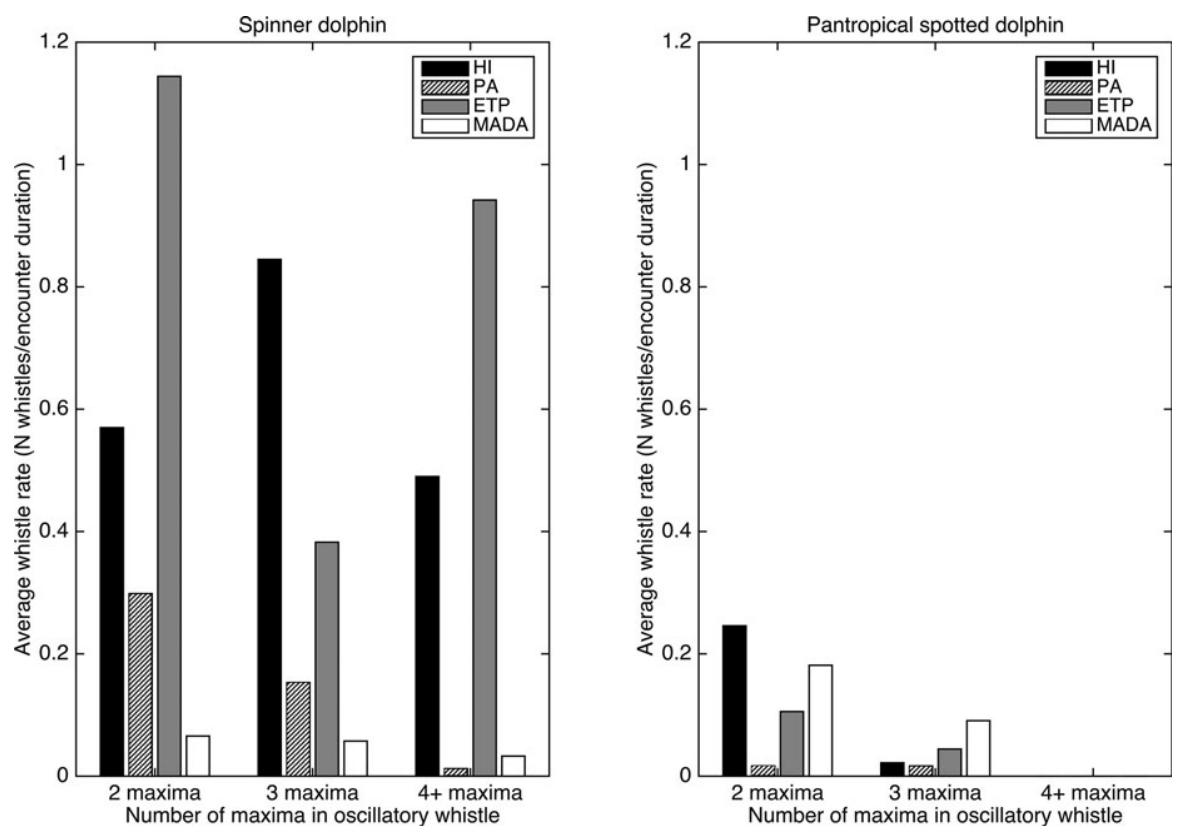

Figure 7. An average whistle rate (number of whistles divided by encounter duration) in spinner (left) and spotted (right) dolphins for each whistle category in each region. 
whistle rates of nonsignature and signature whistles generally increased with increasing group size (Cook et al. 2004). However, for larger groups (above 15 individuals) the signature whistle rate decreased as the group size increased (Quick and Janik 2008). These studies utilized focal follows and signature whistles of individuals and so the exact numbers of vocalizing animals were known. However, since dolphins occur in highly dynamic groups it is not feasible to determine the numbers of actively vocalizing individuals in the context of a line transect survey. In the present study visual observers could only estimate the group size and not the number of vocalizing animals, so given the nontrivial relationship between group size and general whistle rates, it is unsurprising that in this study no clear relationship between observed group size and oscillatory whistle rate could be determined.

When oscillatory whistle rates were compared between species and regions it was observed that rates were higher in spinner than in spotted dolphins in all regions except for Madagascar. It should be noted that while encounter duration for spinner dolphin in this region was longer than encounter duration for spotted dolphin, the number of spinner dolphin encounters was small. The small number of spinner dolphin encounters in Madagascar means that there is little statistical power supporting any conclusions made about this species at this location.

While whistles with four or more maxima were observed only in spinner dolphins, they were found in all four regions investigated: Madagascar, Palmyra, Hawaii, and ETP. This whistle type therefore represents a wide-ranging feature that occurs globally in spinner dolphin populations. A signal shared among different populations and ecotypes has previously been reported in killer whales (Rehn et al. 2011). The V4 call is a universal oscillatory signal with many harmonics and high variation in structure (frequency, sharpness of maxima) but the pattern of frequency modulations is still recognizable as the V4 call (Rehn et al. 2011). Although the oscillatory whistles found in spinner dolphins in this study might not be as distinguishable as the V4 call, the pattern of frequency modulation is recognizable among populations.

Different delphinid species are most commonly classified using whistle frequency contour characteristics such as the start, end, maximum, minimum, and mean frequency, the whistle duration, and the number of inflection points (e.g., Matthews et al. 1999, Rendell et al. 1999, Oswald et.al. 2007). Distinguishing between acoustic signals of small delphinid species (such as the two congeneric species investigated in this paper) is often difficult due to the similar frequency characteristics of their whistles and can result in poor correct classification scores (Oswald et al. 2007). In such cases additional features (whistle characteristics), which could be added as an additional classification rule, could help improve classifier performance. One such additional decision rule could be the presence of oscillatory whistles, despite their low frequency of occurrence (they occurred in $57 \%$ of spinner dolphin encounters and $40 \%$ of spotted dolphin encounters), since they were present in all regions investigated and provided distinction between the two species. Since both species in this study occur in the same habitats, produce whistles with overlapping ranges and exhibit variation in whistle characteristics within the same species between different regions, the identification of global features could improve current classification efforts.

However, in order to efficiently make use of oscillatory whistles as an additional feature, an automated method for tracking of full (unbroken) whistle contours is needed. The majority of fully automated methods applied to odontocete whistle detection and contour extraction are based on spectrogram techniques, where 
following the initial noise removal, spectral maxima are determined at each time step and filtering is performed to extract the relevant maxima. Examples of automated methods include ROCCA (Oswald et al. 2007), Silbido contour extraction software (Roch et al. 2011), adaptive notch-filtering technique (Johansson and White 2011) and PAMGuard whistle detector (Gillespie et al. 2013). Automated methods face many challenges, such as variations in shape, frequency and amplitude of the whistles, varying signal to noise ratio, interfering signals (other whistles and echolocation clicks), varying numbers of whistles at any given time and others. These challenges often result in "breaking" of the contours into multiple detections and prevent the extraction of complete contours, severely limiting the ability to determine the number of maxima in an oscillatory whistle. A potential solution to overcome this problem could be application of multitarget tracking techniques.

This study identified the global presence of oscillatory whistles in two closely related delphinid species. The use of the oscillatory whistles as an additional decision rule in classification could work for single species encounters, where the decision is to be made between spinner and pantropical spotted dolphin and where oscillatory whistles are identified. Future work should investigate the performance of a whistlebased classifier when a decision rule based on oscillatory whistle occurrence is included and further the suitability of oscillatory whistles as features with respect to the whistles of other delphinid species needs consideration.

\section{ACKNOWLEDGMENTS}

The data used in this study came from surveys that were conducted by five different research organizations; Wildlife Conservation Society (WCS); Southwest Fisheries Science Center (NOAA, NMFS); Pacific Islands Fisheries Science Center (NOAA NMFS); Hawaii Institute of Marine Biology, University of Hawaii, and Scripps Institution of Oceanography, University of California, San Diego. We thank all the people involved in the visual and acoustic data collection. We would also like to thank Erin Oleson from NOAA PIFSC, Jay Barlow and Shannon Rankin from NOAA SWFSC for their permission to use the data in the present study.

\section{Literature Cited}

Au, W. W. L. 2000. Hearing in whales and dolphins: An overview. Pages 1-42 in W. W. L. Au, A. N. Popper and R. R. Fay, eds. Hearing by whales and dolphins. Springer-Verlag New York Inc, New York, NY.

Barlow, J., and B. L. Taylor. 2005. Estimates of sperm whale abundance in the northeastern temperate Pacific from a combined acoustic and visual survey. Marine Mammal Science 21:429-455.

Baumann-Pickering, S., S. M. Wiggins, J. A. Hildebrand, M. A. Roch and H. U. Schnitz. 2010. Discriminating features of echolocation clicks of melon-headed whales (Peponocephala electra), bottlenose dolphins (Tursiops truncatus), and Gray's spinner dolphins (Stenella longirostris longirostris). Journal of the Acoustical Society of America 128:2212-2224.

Bazua-Duran, C., and W. W. L. Au. 2002. The whistles of Hawaiian spinner dolphins. Journal of the Acoustical Society of America 112:3064-3072.

Camargo, F. S. Jr, M. M. Rollo, V. Giampaoli and C. Bellini. 2006. Whistle variability in South Atlantic spinner dolphins from the Fernando de Noronha Archipelago off Brazil. Journal of the Acoustical Society of America 120:4071-4079. 
Clark, C. W., and P. J. Clapham. 2004. Acoustic monitoring on a humpback whale (Megaptera novaeangliae) feeding ground shows continual singing into late spring. Proceedings of the Royal Society London B 271:1051-1057.

Cook, M. L. H., L. S. Sayigh, J. E. Blum and R. S. Wells. 2004. Signature-whistle production in undisturbed free-ranging bottlenose dolphins (Tursiops truncatus). Proceedings of the Royal Society London B 271:1043-1049.

Fay, M. P. 2014. rateratio.test: Exact rate ratio test. R package version 1.0-2. Available at http://CRAN.R-project.org/package= rateratio.test.

Gerrodette, T., B. L. Taylor, R. Swift, S. Rankin and A. M. Jaramillo-Legorreta. 2011. A combined visual and acoustic estimate of 2008 abundance, and change in abundance since 1997, for the vaquita, Phocoena sinus. Marine Mammal Science 27:E79-E100.

Gillespie, D., M. Caillat, J. Gordon and P. R. White. 2013. Automatic detection and classification of odontocete whistles. Journal of the Acoustical Society of America 134:2427-2437.

Herzing, D. L. 1996. Vocalizations and associated behaviour of free-ranging Atlantic spotted dolphins, Stenella frontalis and bottlenose dolphins, Tursiops truncatus. Aquatic Mammals 22:61-79.

Janik, V. M., L. S. Sayigh and R. S. Wells. 2006. Signature whistle shape conveys identity information to bottlenose dolphins. Proceedings of the National Academy of Sciences of the United States of America 103:8293-8297.

Johansson, A. T., and P. R. White. 2011. An adaptive filter-based method for robust, automatic detection and frequency estimation of whistles. Journal of the Acoustical Society of America 130:893-903.

King, S. L., and V. M. Janik. 2013. Bottlenose dolphins can use learned vocal labels to address each other. Proceedings of the National Academy of Sciences of the United States of America 110:13216-13221.

Kusel, E. T., D. K. Mellinger, L. Thomas, T. A. Marques, D. Moretti and J. Ward. 2011. Cetacean population density estimation from single fixed sensors using passive acoustics. Journal of the Acoustical Society of America 129:3610-3622.

Lammers, M. O., W. W. L. Au and D. L. Herzing. 2003. The broadband social acoustic signaling behavior of spinner and spotted dolphins. Journal of the Acoustical Society of America 114:1629-1639.

Li, H. Q., M. L. Tang and W. K. Wong. 2013. Confidence intervals for ratio of two Poisson rates using the method of variance estimates recovery. Computational Statistics 29:869889.

Matthews, J. N., L. E. Rendell, J. C. D. Gordon and D. W. MacDonald. 1999. A review of frequency and time parameters of cetacean tonal calls. The International Journal of Animal Sound and its Recording 10:47-71.

May-Collado, L. J., and D. Wartzok. 2008. A comparison of bottlenose dolphin whistles in the Atlantic ocean: Factors promoting whistle variation. Journal of Mammalogy 89:1229-1240.

Mellinger, D. K., K. M. Stafford, S. E. Moore, R. P. Dziak and H. Matsumoto. 2007. An overview of fixed passive acoustic observation methods for cetaceans. Oceanography 20 (4):36-45.

Moore, S. E., K. M. Stafford, D. K. Mellinger and J. A. Hildebrand. 2006. Listening for large whales in the offshore waters of Alaska. BioScience 56:49-55.

Oswald, J. N., J. Barlow and T. F. Norris. 2003. Acoustic identification of nine delphinid species in the Eastern Tropical Pacific Ocean. Marine Mammal Science 19:20-37.

Oswald, J. N., S. Rankin, J. Barlow and M. O. Lammers. 2007. A tool for real-time acoustic species identification of delphinid whistles. Journal of the Acoustical Society of America 122:587-595.

Oswald, J. N., S. Rankin and J. Barlow. 2008. To whistle or not to whistle? Geographic variation in the whistling behaviour of small odontocetes. Aquatic Mammals 34:288302. 
Perrin, W. F. 1975. Distribution and differentiation of populations of dolphins of the genus Stenella in the Eastern Tropical Pacific. Journal of the Fisheries Research Board of Canada 32:1059-1067.

Perrin, W. F., P. A. Akin and J. V. Kashiwada. 1991. Geographic variation in external morphology of the spinner dolphin Stenella longirostris of the Eastern Pacific and implications for conservation. Fishery Bulletin, U.S. 89:411-428.

Psarakos, S., D. Herzing and K. Marten. 2003. Mixed-species associations between Pantropical spotted dolphins (Stenella attenuata) and Hawaiian spinner dolphins (Stenella longirostris) off Oahu, Hawaii. Aquatic Mammals 29:390-395.

Quick, N. J., and V. M. Janik. 2008. Whistle rates of wild bottlenose dolphins (Tursiops truncatus): Influences of group size and behavior. Journal of Comparative Psychology 122:305-311.

Rankin, S., T. F. Norris, M. A. Smultea, C. Oedekoven, A. M. Zoidis, E. Silva and J. Rivers. 2007. A visual sighting and acoustic detections of minke whales, Balenoptera acutorostrata (Cetacea: Balenopteridae), in nearshore Hawaiian waters. Pacific Science 61:395-398.

Rendell, L. E., J. N. Matthews, A. Gill, J. C. D. Gordon and D. W. Macdonald. 1999. Quantitative analysis of tonal calls from five odontocete species, examining interspecific and intraspecific variation. Journal of Zoology (London) 249:403-410.

Reeves, R. R., B. S. Stewart, P. J. Clapham and J. A. Powell. 2002. Guide to marine mammals of the world. Chanticleer Press Inc, New York, NY.

Rehn, N., O. A. Filatova, J. W. Durban and A. D. Foote. 2011. Cross-cultural and crossecotype production of killer whale "excitement" call suggest universality. Naturwissenschaften 98:1-6.

Roch, M. A., M. S. Soldevilla, J. C. Burtenshaw, E. E. Henderson and J. A. Hildebrand. 2007. Gaussian mixture model classification of odontocetes in the Southern California Bight and the Gulf of California. Journal of the Acoustical Society of America 121:1737-1748.

Roch, M. A., T. S. Brandes, B. Patel, Y. Barkley, S. Baumann-Pickering and M. S. Soldevilla. 2011. Automated extraction of odontocete whistle contours. Journal of the Acoustical Society of America 130:2212-2223.

Selvin, S. 2004. Statistical analysis of epidemiological data. Chapter 9. Third edition. Oxford University Press, Inc., New York, NY.

Shirihai, H. 2006. Whales, dolphins and seals. A\&C Black Publishers Ltd., London, U.K.

Soldevilla, M. S., E. E. Henderson, G. S. Campbell, S. M. Wiggins, J. A. Hildebrand and M. A. Roch. 2008. Classification of Risso's and Pacific white-sided dolphins using spectral properties of echolocation clicks. Journal of the Acoustical Society of America 124:609624.

Tyack, P. L., and C. W. Clark. 2000. Communication and acoustic behaviour of dolphins and whales. Pages 156-224 in W. W. L. Au, A. N. Popper and R. R. Fay, eds. Hearing by whales and dolphins. Springer-Verlag New York Inc, New York, NY.

Van Parijs, S. M., C. W. Clark, R. S. Sousa-Lima, S. E. Parks, S. Rankin, D. Risch and I. C. Van Opzeeland. 2009. Management and research applications of real-time and archival passive acoustic sensors over varying temporal and spatial scales. Marine Ecology Progress Series 395:21-36. 